\title{
ON UNIQUENESS IN THE EXTENDED SELBERG CLASS OF DIRICHLET SERIES
}

\author{
HASEO KI AND BAO QIN LI \\ (Communicated by Mario Bonk)
}

\begin{abstract}
We will show that two functions in the extended Selberg class satisfying the same functional equation must be identically equal if they have sufficiently many common zeros.
\end{abstract}

This paper concerns the question of how $L$-functions are determined by their zeros. $L$-functions are Dirichlet series with the Riemann zeta function $\zeta(s)=$ $\sum_{n=1}^{\infty} \frac{1}{n^{s}}$ as the prototype and are important objects in number theory. The Selberg class $\mathcal{S}$ of $L$-functions is the set of all Dirichlet series $L(s)=\sum_{n=1}^{\infty} \frac{a(n)}{n^{s}}$ of a complex variable $s=\sigma+i t$ with $a(1)=1$, satisfying the following axioms (see [7]):

(i) (Dirichlet series) For $\sigma>1, L(s)$ is an absolutely convergent Dirichlet series.

(ii) (Analytic continuation) There is a nonnegative integer $k$ such that $(s-$ $1)^{k} L(s)$ is an entire function of finite order.

(iii) (Functional equation) $L$ satisfies a functional equation of type

$$
\Lambda_{L}(s)=\omega \overline{\Lambda_{L}(1-\bar{s})},
$$

where $\Lambda_{L}(s)=L(s) Q^{s} \prod_{j=1}^{K} \Gamma\left(\lambda_{j} s+\mu_{j}\right)$ with positive real numbers $Q, \lambda_{j}$ and with complex numbers $\mu_{j}, \omega$ with $\operatorname{Re} \mu_{j} \geq 0$ and $|\omega|=1$.

(iv) (Ramanujan hypothesis) $a(n) \ll n^{\varepsilon}$ for every $\varepsilon>0$;

(v) (Euler product) $\log L(s)=\sum_{n=1}^{\infty} \frac{b(n)}{n^{s}}$, where $b(n)=0$ unless $n$ is a positive power of a prime and $b(n) \ll n^{\theta}$ for some $\theta<\frac{1}{2}$.

The degree $d_{L}$ of an $L$-function $L$ is defined to be $d_{L}=2 \sum_{j=1}^{K} \lambda_{j}$, where $K, \lambda_{j}$ are the numbers in axiom (iii).

The Selberg class includes the Riemann zeta-function $\zeta$ and essentially those Dirichlet series where one might expect the analogue of the Riemann hypothesis. At the same time, there are a whole host of interesting Dirichlet series not possessing a Euler product (see e.g. 3, 8]). Throughout the paper, all $L$-functions are assumed to be functions from the extended Selberg class of those only satisfying the axioms (i)-(iii) (see [3]). Thus, the results obtained in the present paper particularly apply to $L$-functions in the Selberg class.

Received by the editors October 5, 2011 and, in revised form, February 12, 2012.

2010 Mathematics Subject Classification. Primary 11M36, 30D30.

Key words and phrases. L-function, Dirichlet series, Selberg class, uniqueness.

The first named author was supported by the Mid-career Researcher Program through an NRF grant funded by the MEST 2010-0008706. 
It was pointed out in [1] that two $L$-functions are expected to have few common zeros if they are "independent", and this problem appears to be very difficult (see [1] for the details and for the meaning of independence). On the other hand, $L$ functions are meromorphic functions, and meromorphic functions possess the wellknown uniqueness property by Nevanlina's uniqueness theorem: two nonconstant meromorphic functions $f, g$ in $\mathbf{C}$ must be identically equal if $f$ and $g$ share five distinct values $c_{j} \in \mathbf{C} \cup\{\infty\}$ in the sense that $f-c_{j}$ and $g-c_{j}$ have the same zeros without counting multiplicities (see e.g. [2 or [8]). It is known that two $L$-functions are equal if they share a value with counting multiplicities ([8, p. 152]). For $L$-functions satisfying the same functional equation and sharing two complex numbers $c_{1}, c_{2}$, Steuding proved the uniqueness under a condition on the number of the distinct zeros of $L-c_{j}$ (see [8, p. 152]). Li ([5]) recently removed this condition. Ki (4]) further considered if this still holds for one shared value and, in particular, showed that two $L$-functions $L_{1}, L_{2}$ satisfying the same functional equation can be distinct if $L_{1}, L_{2}$ have the same zeros with different multiplicities. This however cannot happen if the multiplicity of a zero of $L_{1}$ does not exceed that of $L_{2}$ allowing some exceptions ([6]). In the present paper, we show that an $L$-function $L$ is completely determined by the functional equation and its nontrivial zeros $\rho$, allowing an exceptional set $G$ of $\rho$ with the largest possible size. As usual, the nontrivial zeros of an $L$-function are the zeros which are not located at the poles of the $\Gamma$ factors in the functional equation. Denote by $Z^{+}(L)$ the set of all nontrivial zeros of $L$ counted with multiplicity. The size of $G$ is measured by the usual counting function $n(r, G)$, the number of points in $G \cap\{|s|<r\}$ counted with multiplicity $(n(r, G)=0$ if $G$ is empty). Since $n(r, Z(L))$ of the zero set $Z(L)$ of a degree zero $L$-function $L$ is $O(r)$, it would be tempting to think that the condition $n(r, G)=o(r)$ would be the best to obtain for the exceptional set. However, a quite delicate analysis shows that a sharp condition can be given in terms of the type of $G$, which is defined as

$$
\tau(G):=\limsup _{r \rightarrow \infty} \frac{n(r, G)}{r} .
$$

More precisely, we have the following:

Theorem. Two nonconstant functions $L_{1}$ and $L_{2}$ in the extended Selberg class satisfying the same functional equation are identically equal if $Z^{+}\left(L_{1}\right) \backslash G \subseteq Z^{+}\left(L_{2}\right)$ for a set $G$ satisfying that $\tau(G)<\frac{\log 4}{\pi}$. Furthermore, this inequality for $\tau(G)$ is best possible.

We note that "the same functional equation" condition in the theorem is crucial and cannot be dropped. For example, $\zeta$ and $\zeta^{2}$ clearly satisfy that $Z^{+}(\zeta) \subset Z^{+}\left(\zeta^{2}\right)$, but they are not equal.

Proof. Consider the auxiliary function

$$
f(s):=\left(s^{2}-1\right)^{m} s^{n} \frac{L_{2}(s)-L_{1}(s)}{L_{1}(s)} \frac{L_{2}(-s)-L_{1}(-s)}{L_{1}(-s)} \prod_{\rho \in G}\left(1-\frac{s^{2}}{\rho^{2}}\right) .
$$

It is easy to check that the infinite product in (1) converges to an entire function (cf. (2) below). Since $L_{1}, L_{2}$ satisfy the same functional equation, $L_{1}-L_{2}$ satisfies the same functional equation. Thus, $L_{1}$ and $L_{1}-L_{2}$ have the same trivial zeros that are located at the poles of the $\Gamma$ factors in the functional equation. These zeros do not produce any poles of $f$ due to cancellation. Hence, there are integers $m, n$ such 
that the function $f$ is an entire function (we may then assume that $s=0$ is not in $G)$. We may increase $m$ so that $s= \pm 1$ are zeros of $f$. Choose $0<D_{0}<D_{1}<1$ with $\tau(G)<D_{0} \frac{\log 4}{\pi}$. Then, there is an $r_{0}>0$ such that $\frac{n(r, G)}{r}<D_{0} \frac{\log 4}{\pi}$ for $r \geq r_{0}$. We deduce that for large $|s|$,

$$
\begin{aligned}
& \log \left|\prod_{\rho \in G}\left(1-\frac{s^{2}}{\rho^{2}}\right)\right| \leq \sum_{\rho \in G} \log \left(1+\left|\frac{s^{2}}{\rho^{2}}\right|\right) \\
& =\int_{0}^{\infty} \log \left(1+\frac{\left|s^{2}\right|}{r^{2}}\right) d n(r, Z(G)) \\
& =2|s|^{2} \int_{0}^{\infty} \frac{n(r, Z(G))}{r\left(r^{2}+|s|^{2}\right)} d r \\
& \leq 2|s|^{2}\left\{\frac{1}{|s|^{2}} \int_{0}^{r_{0}} \frac{n(r, Z(G))}{r} d r+D_{0} \frac{\log 4}{\pi} \int_{r_{0}}^{\infty} \frac{1}{r^{2}+|s|^{2}} d r\right\} \\
& \leq D_{1}|s| \log 4 .
\end{aligned}
$$

Dividing the functional equation of $L_{1}-L_{2}$ by the same functional equation of $L_{1}$, we obtain that

$$
\frac{L_{2}(s)-L_{1}(s)}{L_{1}(s)}=\frac{\overline{L_{2}(1-\bar{s})}-\overline{L_{1}(1-\bar{s})}}{\overline{L_{1}(1-\bar{s})}} .
$$

Noting that $L_{1}(s)=\sum_{n=1}^{\infty} \frac{a(n)}{n^{s}}$ with $a(1)=1$, we have that

$$
L_{1}(s) \rightarrow 1 \text { and } L_{1}(s)-L_{2}(s)=O\left(\frac{1}{2^{\sigma}}\right) \quad(\sigma \rightarrow+\infty) .
$$

This together with (3) yields that

$$
\left|\frac{L_{2}(s)-L_{1}(s)}{L_{1}(s)} \cdot \frac{L_{2}(-s)-L_{1}(-s)}{L_{1}(-s)}\right|=O\left(\frac{1}{4^{|\sigma|}}\right)
$$

as $\sigma \rightarrow \pm \infty$. Therefore, by (1) and (2) we have that for $D$ with $D_{1}<D<1$,

$$
\log |f(s)| \leq D|s| \log 4-|\sigma| \log 4+O(1) \quad(\sigma \rightarrow \pm \infty) .
$$

It is then easy to see that $f$ is bounded on the rays $\arg (s)=\theta, \pi-\theta, \pi+\theta, 2 \pi-\theta$, where $0<\theta<\pi / 2$ with $\cos \theta=D$, since on the rays, $|\cos \theta|=\frac{|\sigma|}{|s|}=D$. Note that nonconstant $L$-functions are of order 1 (see [8, p. 150], 9]) and the infinite product in (1) is also of order 1 . Thus, $f$ is of order at most 1 . We then have that $f(s)=O\left(e^{|s|^{1+\epsilon}}\right)$ for any $\epsilon>0$. Hence, $f$ satisfies the conditions of the PhragménLindelöf theorem in each of the sectors bounded by the above rays, and thus $f$ is bounded in each of the sectors and thus in the entire complex plane. Therefore the entire function $f$ must be a constant. But $f$ has a zero. Thus $f$ and then $L_{1}-L_{2}$ must be identically zero.

It remains to prove that the inequality $\tau(G)<\frac{\log 4}{\pi}$ is best possible. To this end, consider the following two $L$-functions:

$$
l_{1}(s)=1+\frac{2}{4^{s}}, \quad l_{2}(s)=1+\frac{1}{2^{s}}+\frac{2}{4^{s}} .
$$

Then one can verify that for $j=1,2$,

$$
2^{s} l_{j}(s)=2^{1-s} \overline{l_{j}(1-\bar{s})} .
$$


Thus, $l_{1}, l_{2}$ are $L$-functions of degree 0 satisfying the same functional equation. Recall the Riemann-von-Mangoldt formula for $L$-functions (see [8, p. 145]): for a nonconstant $L$-function $L$ of degree 0 ,

$$
N_{L}^{0}(T)=\frac{T}{\pi} \log \left(Q^{2}\right)+O(\log T),
$$

where $Q$ is the number in the functional equation in axiom (iii) and $N_{L}^{0}(T)$ denotes the number of zeros (counting multiplicities) of $L$ in the region $\operatorname{Re} s>0,|\operatorname{Im} s| \leq T$. Thus,

$$
\frac{n\left(r, Z\left(l_{1}\right)\right)}{r} \sim \frac{\log 4}{\pi}
$$

as $r \rightarrow \infty$. Set $L_{1}=L l_{1}$ and $L_{2}=L l_{2}$, where $L$ is any $L$-function (of arbitrarily given degree). Then $L_{1}, L_{2}$ satisfy the same functional equation. Now, take the exceptional set $G$ to be the entire set $Z\left(l_{1}\right)$. Then $G$ satisfies that $\tau(G)=\frac{\log 4}{\pi}$, and it is clear that $Z^{+}\left(L_{1}\right) \backslash G \subset Z^{+}\left(L_{2}\right)$. However, $L_{1} \neq L_{2}$. This proves the theorem.

We conclude the paper by noting that the above theorem is clearly related to the question of when a functional equation in axiom (iii) has a unique $L$-function solution. Let $L$ be an $L$-function solution of the functional equation

$$
Q^{s} L(s)=\omega Q^{1-s} \overline{L(1-\bar{s})} .
$$

Then

$$
\frac{n(r, Z(L))}{r} \sim \frac{\log Q^{2}}{\pi}
$$

by the Riemann-von-Mangoldt formula (cf. the proof of the Theorem). Thus, when $Q^{2}<4$ we can take the entire set $Z(L)$ as an exceptional set, which, by the theorem, implies that two $L$-functions satisfying the same functional equation with $0<Q<2$ must be equal; i.e., $L$ is the unique solution. The inequality $0<Q<2$ is best possible, since the uniqueness breaks down when $Q=2$, as seen in the proof of the Theorem for the functional equation

$$
2^{s} L(s)=2^{1-s} \overline{L(1-\bar{s})},
$$

which has two distinct $L$-function solutions.

\section{ACKNOWLEDGEMENT}

The authors thank the referee for valuable suggestions.

\section{REFERENCES}

[1] E. Bombieri and A. Perelli, Distinct zeros of L-functions, Acta Arith. 83 (1998), no. 3, 271281. MR1611193 (99a:11102)

[2] W. K. Hayman, Meromorphic functions, Oxford Mathematical Monographs, Clarendon Press, Oxford, 1964. MR0164038 (29 \#1337)

[3] Jerzy Kaczorowski and Alberto Perelli, On the structure of the Selberg class. I. $0 \leq d \leq 1$, Acta Math. 182 (1999), no. 2, 207-241, DOI 10.1007/BF02392574. MR.1710182(2000h:11097)

[4] H. Ki, Uniqueness of functions in the extended Selberg class, preprint.

[5] Bao Qin Li, A uniqueness theorem for Dirichlet series satisfying a Riemann type functional equation, Adv. Math. 226 (2011), no. 5, 4198-4211, DOI 10.1016/j.aim.2010.12.001. MR.2770446(2012c:11190) 
[6] Bao Qin Li, On common zeros of L-functions, Math. Z. 272 (2012), no. 3-4, 1097-1102, DOI 10.1007/s00209-011-0977-5. MR2995159

[7] Atle Selberg, Old and new conjectures and results about a class of Dirichlet series, Proceedings of the Amalfi Conference on Analytic Number Theory (Maiori, 1989), Univ. Salerno, Salerno, 1992, pp. 367-385. MR.1220477 (94f:11085)

[8] Jörn Steuding, Value-distribution of L-functions, Lecture Notes in Mathematics, vol. 1877, Springer, Berlin, 2007. MR2330696 (2008m:11172)

[9] Zhuan Ye, The Nevanlinna functions of the Riemann Zeta-function, J. Math. Anal. Appl. 233 (1999), no. 1, 425-435, DOI 10.1006/jmaa.1999.6343. MR.1684396 (2000d:30047)

Department of Mathematics, Yonsei University, Seoul 120-749, Republic of Korea E-mail address: haseo@yonsei.ac.kr

Department of Mathematics, Florida International University, Miami, Florida 33199

E-mail address: libaoqin@fiu.edu 